\title{
AVALIAÇÃO TRANSVERSAL DOS MÉTODOS TERAPÊUTICOS DO PÉ TORTO CONGÊNITO EQUINOVARO IDIOPÁTICO: CONTROVÉRSIAS SOBRE A TENOTOMIA DO TENDÃO CALCÂNEO
}

\author{
CROSS-EVALUATION OF THE THERAPEUTICAL METHODS FOR IDIOPATHIC \\ CONGENITAL CLUBFOOT (TALIPES EQUINOVARUS): CONTROVERSIES \\ REGARDING THE TENDOCALCANEOUS TENOTOMY
}

\author{
Renan Moukbel Chaim', Fabio Assunção e Silva', Felipe Bertelli Angelini', Eiffel Tusuyoshi Dobashi², \\ Luiz Carlos de Andrade Júnior ${ }^{3}$, Francesco Camara Blumetti' ${ }^{4}$, José Antonio Pinto ${ }^{5}$
}

\section{RESUMO}

Objetivo: Há muita discussão em relação ao tratamento do pé torto congênito equino-cavo-varo e atualmente a sistemática proposta por Ponseti parece ser a mais racional oferecendo altas taxas de resultados satisfatórios em detrimento à metodologia de Kite que predominou no meio ortopédico até o final da década de 90 . Com a recente mudança de conceitos, este trabalho tem o intuito de analisar, por meio de questionário aplicado no $39^{\circ}$ Congresso Brasileiro de Ortopedia e Traumatologia, o perfil dos ortopedistas no Brasil frente a esta afecção, fato este desconhecido considerando a literatura nacional. Método: Um questionário para investigação foi elaborado para verificar o método de tratamento utilizado, as características da população estudada, os resultados obtidos com o tratamento e particularmente como abordam cirurgicamente o tendão calcâneo. Resultados: Do total de 5.329 ortopedistas inscritos, obtivemos 539 participações espontâneas; destes, somente $88(16,30 \%)$ indivíduos executam o tratamento para o pé torto congênito; $78(88,60 \%)$ aplicam o método de Ponseti; e nove (10,20\%) o de Kite. Para 47,70\%, o tratamento conservador é realizado entre quatro e seis meses e para $35,20 \%$ entre um e três meses; $58(66,00 \%)$ entrevistados efetuam a tenotomia do tendão calcâneo em $80 \%$ a $100 \%$ de seus pacientes e $59(67,05 \%)$ a realizam no centro cirúrgico devido às condições de segurança, assepsia, anestesia, facilidade de acesso e monitorização do paciente; 32 (36,36\%) ortopedistas apresentam $80 \%$ ou mais de bons resultados, $54(61,36 \%)$ apresentam $50 \%$ a $80 \%$ de bons resultados e $46(52,27 \%)$ apresentam taxa

\section{ABSTRACT}

Objective: There has been a lot of discussion regarding the treatment of congenital clubfoot (talipes equinovarus,) and Posenti's methodology currently seems to be the most rational, offering high rates of satisfactory results when compared to Kite's approach that prevailed in orthopedics until the end of the 90s. With the recent change of concepts, this study purports to analyze the profile of orthopedists treating this infirmity in Brazil, through a questionnaire used at the 39th Brazilian Congress of Orthopaedics and Traumatology, since such data is unknown in the Brazilian literature. Methods: An investigative questionnaire was prepared to ascertain the treatment method used, the characteristics of the population studied, the results acquired with treatment and, particularly, how they approached the Achilles tendon. Results: Of the 5,329 registered orthopedists, we acquired 539 spontaneous participations. Of these, only 88 $(16.30 \%)$ orthopedists perform the treatment for congenital clubfoot; 78 (88.60\%) use the Ponseti method and 9 (10.20\%) use Kite's; for $47.70 \%$, conservative treatment is performed between 4 and 6 months and for $35.30 \%$, between 1 and 3 months; 58 $(66.00 \%)$ interviewees perform the Achilles tendon tenotomy in $80 \%$ to $100 \%$ of their patients and $59(67.05 \%)$ perform it at a surgical center due to safety conditions, sterilized environment, anesthesia, ease of access, and patient monitoring; 32 (36.36\%) orthopedists present $80 \%$ of good results or more, 54 (61.36\%) present $50 \%$ to $80 \%$ good results and $46(52.27 \%)$ present a 10\% relapse rate. Conclusions: Although the Ponseti Method

1 - Médico Residente do Departamento de Ortopedia e Traumatologia da Universidade Federal de São Paulo - São Paulo, Brasil.

2 - Doutor, Chefe de Clínica da Disciplina de Ortopedia Pediátrica do Departamento de Ortopedia e Traumatologia da Universidade Federal de São Paulo São Paulo, Brasil.

3 - Mestre pela Disciplina de Ortopedia Pediátrica do Departamento de Ortopedia e Traumatologia da Universidade Federal de São Paulo - São Paulo, Brasil.

4 - Médico Assistente da Disciplina de Ortopedia Pediátrica do Departamento de Ortopedia e Traumatologia da Universidade Federal de São Paulo - São Paulo, Brasil.

5 - Professor Adjunto, Chefe da Disciplina de Ortopedia Pediátrica do Departamento de Ortopedia e Traumatologia da Universidade Federal de São Paulo São Paulo, Brasil.

Trabalho desenvolvido na Disciplina de Ortopedia Pediátrica do Departamento de Ortopedia e Traumatologia da Universidade Federal de São Paulo Escola Paulista de Medicina.

Correspondência: Felipe Bertelli Angelini - Rua Borges Lagoa, 783 - 5ªndar - Vila Clementino - São Paulo, SP. E-mail: fi_angelini@hotmail.com 
de recidiva de $10 \%$. Conclusão: Embora o método de Ponseti defina que a tenotomia do tendão calcâneo seja realizada ambulatorialmente com anestesia local, a maioria 59 (67,05\%) dos ortopedistas a realiza no centro cirúrgico.

Descritores - Pé Torto; Questionários; Tendão do Calcâneo; Método

\section{INTRODUÇÃO}

O pé torto congênito (PTC) idiopático equino-cavovaro é reconhecido desde a Antiguidade e, ao longo do tempo, muitos trabalhos científicos foram desenvolvidos. A pesquisa em base de dados Medline aponta que, entre 1966 e 2008, foram desenvolvidos 2.244 artigos versando sobre este assunto. Particularmente, quando abordamos o tratamento, observamos propostas terapêuticas diferentes e que, até o presente momento, apresentam controvérsias quanto à melhor opção. A variedade de técnicas pode ser atribuída à dificuldade na execução do tratamento, aos diferentes graus de manifestação da doença e a experiência individual de cada cirurgião com relação as técnicas aplicadas.

A partir da segunda metade do século XX, os métodos mais popularizados foram: o de $\mathrm{Kite}^{(1)} \mathrm{e}$, atualmente, o preconizado por Ponseti ${ }^{(2)}$, que tem se destacado por demonstrar altos índices de resultados satisfatórios. Esta sistemática tem como objetivo obter a correção progressiva das deformidades pela aplicação de aparelhos gessados seriados, sucedida pela tenotomia percutânea do tendão calcâneo. Faz parte integrante do método ${ }^{(3)} \mathrm{o}$ alongamento do tendão calcâneo pela abordagem percutânea com o intuito de obter-se a correção do equinismo. Entretanto, pelo risco de potenciais complicações da abordagem percutânea como, por exemplo, a lesão da artéria fibular $^{(4)}$, encontramos cirurgiões que defendem e estimulam a opção pelas tenotomias realizadas pela abordagem aberta.

Atualmente, a sistemática proposta por Ponseti $^{(2)}$ parece ser a mais racional, oferecendo potencialmente os melhores resultados clínicos de acordo com a literatura mundial. Com esta recente mudança de conceitos, este trabalho foi elaborado com o objetivo de avaliar como os ortopedistas abordam terapeuticamente o PTC idiopático por meio de questionário, fato este desconhecido considerando a literatura nacional. Há polêmica em relação à necessidade e ao método utilizado para realizar o alongamento do tendão calcâneo que participa da metódica de correção, enfatizando qual é a abordagem cirúrgica preferencial (via aberta ou percutânea). defines that the Achilles tendon tenotomy should be performed in an outpatient setting, most of the orthopedists (59-67.05\%) perform it in the operating room.

Keywords - Clubfoot; Questionnaires; Achilles Tendon; Methods

\section{MATERIAL E MÉTODO}

Inicialmente, este trabalho foi submetido à apreciação do Comitê de Ética Médica em Pesquisa e aprovado para realização sob o número 0282/08.

Elaborou-se um protocolo de pesquisa especialmente para este fim que foi direcionado aos médicos ortopedistas que participaram do $39^{\circ} \mathrm{CBOT}$ realizado em São Paulo entre 14 e 17 de novembro de 2007. A opção de tomar parte deste trabalho coube exclusivamente à aceitação espontânea dos participantes; entretanto, consideramos para esta pesquisa somente ortopedistas, excluindo médicos residentes, fisiatras, estrangeiros e profissionais de outras áreas de atuação. O questionário consistiu de uma série de perguntas cujas respostas foram predefinidas pelos autores. Consideramos os aspectos gerais da população analisada como gênero, idade, naturalidade, possuir título de especialista em ortopedia e traumatologia, atuar em uma subespecialidade ortopédica, método de tratamento utilizado e meios de atualização. Uma questão controversa está relacionada com o método de alongamento do tendão calcâneo pela tenotomia para a correção do equino do retropé, logo, elaboramos questões específicas sobre esta abordagem.

Com relação ao tratamento conservador, consideramos o tempo médio da duração do tratamento, utilização de radiografias e classificações para o tratamento e a porcentagem média de bons resultados.

As perguntas referentes ao tratamento cirúrgico abordaram a frequência com que as operações são realizadas, a faixa etária média da população submetida a essa intervenção e as complicações mais frequentemente encontradas.

Para os entrevistados que utilizam o método de Ponseti $^{(2)}$ como modalidade preferencial de tratamento, questionamos: a porcentagem em que a tenotomia do tendão calcâneo é realizada; se a tenotomia é executada no centro cirúrgico ou no ambulatório; e qual a justificativa para tal opção; se os mesmos fazem a opção pela utilização do aparelho de Denis-Brown na fase pósoperatória e se reconhecem dificuldades para o uso e aderência à órtese; quando avaliam seus resultados qual 
a porcentagem de recidivas. Obtivemos uma amostra significante que constou de 539 (10,11\%) indivíduos de um total de 5.329 participantes e desconsideramos a opinião de ortopedistas estrangeiros, médicos residentes, alunos da graduação e profissionais de outras áreas que participaram do Congresso. Em relação ao sexo, observamos 498 (92,39\%) homens e 41 (7,61\%) mulheres. Considerando a região geográfica de origem do entrevistado, obtivemos $492(91,3 \%)$ provenientes do sudeste, $19(3,5 \%)$ do nordeste, $14(2,6 \%)$ do sul, $11(2 \%)$ do norte e três $(0,6 \%)$ do centro-oeste. Desta amostra, 50 (9,3\%) não possuem título de especialista da SBOT e 489 (90,7\%) o possuem. Foram entrevistados 96 (17,8\%) indivíduos com subespecialidade na área da cirurgia do joelho, 84 $(15,6 \%)$ do quadril, $84(15,6 \%)$ da mão, $82(15,2 \%)$ do trauma, $38(7,1 \%)$ em pediátrica, $30(5,6 \%)$ em ombro e cotovelo, $20(3,7 \%)$ de coluna, oito $(1,5 \%)$ em fixadores externos, seis $(1,1 \%)$ em pé, seis $(1,1 \%)$ em medicina esportiva, $62(11,5 \%)$ sem subespecialidade definida e $23(4,2 \%)$ atuam em mais de uma subespecialidade. Do total, $41(7,6 \%)$ são membros da Sociedade Brasileira de Ortopedia Pediátrica e 498 (92,4\%) não.

A análise estatística do material foi realizada por profissional especializado nesta área de atuação e, inicialmente, todas as variáveis foram analisadas descritivamente. Para as variáveis qualitativas calcularam-se frequências absolutas e relativas. Para se testar a homogeneidade entre as proporções foi utilizado o teste do Qui-quadrado $^{(5)}$ e o teste exato de Fisher apud Rosner ${ }^{(5)}$ (quando ocorreram frequências esperadas menores de cinco). Em todos os casos, o nível de significância para rejeição da hipótese de nulidade foi fixado em 0,05 (5\%). Quando a estatística calculada apresentou significância, usamos um asterisco (*) para caracterizá-la.

\section{RESULTADOS}

Desta amostra, observamos que $451(83,7 \%)$ ortopedistas não realizam tratamento para o PTC. Dos 88 $(16,3 \%)$ que executam a terapêutica, $78(88,6 \%) *$ têm preferência pelo método de Ponseti ${ }^{(2)}$ como método de eleição e nove $(10,2 \%)$ optam pela metodologia de Kite $^{(1)}$ (Tabela 1).

Foi observado que, para 47,7\% dos entrevistados, a duração do tratamento conservador ocorre entre quatro e seis meses, e para $35,3 \%$ destes entre um e três meses (Tabela 2).

Verificamos, também, que $63,6 \%$ * não utilizam radiografias para diagnóstico e durante o acompanhamento terapêutico desta entidade nosológica.
Tabela 1 - Preferência por método conservador para tratamento do pé torto congênito - Fisher's Exact Test (2-Tail) 0,053.

\begin{tabular}{c|c|c|}
\hline Método & Indivíduos & Porcentagem (\%) \\
\hline Kite & 9 & 10,2 \\
\hline Ponseti & 78 & 88,66 \\
\hline Outro método & 1 & 1,1 \\
\hline Total & 88 & 100 \\
\hline
\end{tabular}

Tabela 2 - Distribuição do intervalo em meses da duração do tratamento conservador - Chi-Square 0,004.

\begin{tabular}{c|c|c}
\hline Tempo (meses) & Indivíduos & Porcentagem (\%) \\
\hline 01/mar & 31 & 35,3 \\
\hline 04/jun & 42 & 47,7 \\
\hline$>6$ & 15 & 17 \\
\hline Total & 88 & 100 \\
\hline
\end{tabular}

Com relação à realização da tenotomia do tendão calcâneo, $58(66,00 \%)$ ortopedistas efetuam este procedimento em uma proporção entre $80 \%$ e $100 \%$ de seus casos tratados, nos quais, do total $13(14,77 \%)$, a executam em todos $(100 \%)$ os pacientes (Tabela 3$)$. Destes, $59(67,05 \%) *$ realizam este procedimento operatório no centro cirúrgico devido às condições de segurança, assepsia, anestesia, monitorização do paciente, facilidade de acesso, entre outras (Tabela 4).

Tabela 3 - Distribuição quanto à necessidade de realização de tenotomia do tendão calcaneano - Fisher's Exact Test (2-Tail) 0,024.

\begin{tabular}{c|c|c}
\hline Tenotomia (\% de casos operados) & Indivíduos & Porcentagem (\%) \\
\hline$<50 \%$ & 9 & 10,2 \\
\hline $50 \%-80 \%$ & 21 & 23,8 \\
\hline $80 \%-100 \%$ & 58 & 66 \\
\hline Total & 88 & 100 \\
\hline
\end{tabular}

Tabela 4 - Distribuição segundo a realização da tenotomia do tendão calcaneano no centro cirúrgico ou em ambiente ambulatorial - ChiSquare 0,001 .

\begin{tabular}{c|c|c}
\hline Local & Indivíduos & Porcentagem (\%) \\
\hline Ambulatório & 29 & 32,95 \\
\hline Centro cirúrgico & 59 & 67,05 \\
\hline Total & 88 & 100 \\
\hline
\end{tabular}

Dos indivíduos questionados, independente do método de classificação preferencial que utilizam para avaliar os resultados com o tratamento conservados ${ }^{(6-8)}, 32$ (36,36\%)* apresentam $80 \%$ de resultados satisfatórios e $54(61,36 \%) *$ apresentam entre $50 \%$ e $80 \%$ de resultados satisfatórios (Tabela 5). A recidiva é reconhecida 
em até $10 \%$ dos pacientes por $46(52,27 \%)$ ortopedistas entrevistados (Tabela 6).

Tabela 5 - Distribuição dos resultados obtidos pelo método conservador - Fisher's Exact Test (2-Tail) 0,003.

\begin{tabular}{c|c|c}
\hline $\begin{array}{c}\text { Avaliação final } \\
\text { (\% de bons resultados) }\end{array}$ & Indivíduos & Porcentagem (\%) \\
\hline$<50 \%$ & 2 & 2,28 \\
\hline $50 \%$ a $80 \%$ & 54 & 61,36 \\
\hline$>80 \%$ & 32 & 36,36 \\
\hline Total & 88 & 100 \\
\hline
\end{tabular}

Tabela 6 - Distribuição dos indivíduos conforme a frequência de recidivas da deformidade após o tratamento - Fisher's Exact Test (2-Tail) 0,193.

\begin{tabular}{c|c|c}
\hline $\begin{array}{c}\text { Recidivas } \\
\text { (\% de casos) }\end{array}$ & Indivíduos & Porcentagem (\%) \\
\hline$<10 \%$ & 8 & 9,09 \\
\hline $1 \%$ a $10 \%$ & 46 & 52,27 \\
\hline $11 \%$ a $30 \%$ & 23 & 26,12 \\
\hline $31 \%$ a $50 \%$ & 1 & 1,26 \\
\hline$>51 \%$ & 10 & 11,26 \\
\hline Total & 88 & 100 \\
\hline
\end{tabular}

\section{DISCUSSÃO}

O PTC caracteriza-se por ser uma entidade complexa de difícil correção cujo objetivo terapêutico é o de reduzir ou eliminar as deformidades que o caracterizam, permitindo ao paciente acometido alcançar o máximo de sua função motora. Consequentemente, o segmento tratado deve apresentar apoio plantígrado, boa mobilidade, prescindir de áreas de hiperpressão e calosidades, permitir o uso de calçado convencional sem adaptações e estar desprovido da dor.

Os métodos para o tratamento desta afecção estão bem estabelecidos e são amplamente divulgados no tirocínio desta enfermidade. Realizamos uma pesquisa na base de dados Medline (1966 a 2008) dos artigos indexados utilizando o unitermo pé equinovaro e, consequentemente, obtivemos um total de 2.244 trabalhos, sendo que 418 referem-se ao tratamento; destes, 57 abordam o tratamento conservador e nove citam o método de Ponseti ${ }^{(1)}$. Entretanto, desconhecemos a opinião dos ortopedistas brasileiros em relação às metodologias preferencialmente praticadas, os resultados obtidos, as complicações observadas e as modificações e adaptações adotadas quando instituídos tais tratamentos. $\mathrm{Na}$ literatura pesquisada, observamos somente um artigo com objetivo semelhante. A avaliação do perfil do médico ortopedista brasileiro voltado para o tratamento desta deformidade permite-nos estudar qual o status do atendimento desta condição patológica e reconhecer as características da população de médicos pelo instrumento deste estudo transversal.

Desconsiderando o tratamento escolhido, a maioria dos estudos enfoca que a abordagem inicial deve ser não operatória, independente do nível de gravidade observada desde a primeira avaliação. Com relação aos métodos descritos, o preconizado por Ponseti ${ }^{(2)}$ tem demonstrado ser o mais efetivo para a correção para a maioria dos pés tratados, e é, atualmente, a técnica de maior difusão no meio ortopédico, suplantando os demais meios. Na nossa casuística, observamos uma concordância neste quesito de acordo com os resultados da Tabela 1. Outrossim, verificamos que, atualmente, apenas $10,2 \%$ dos entrevistados têm aplicado o método de Kite ${ }^{(1)}$, apontando para uma tendência que corrobora a literatura atual ${ }^{(9,10)}$. Dos nove artigos que se referem ao tratamento pelo método de Ponseti ${ }^{(2)}$, oito foram publicados entre 2002 e 2007.

Quando abordamos a duração do tratamento, constatada na Tabela 2, percebemos que, pela opinião dos ortopedistas, aparentemente, houve diminuição no tempo de uso do aparelho gessado (em torno de quatro a seis meses), comparado os resultados de nossa pesquisa com os valores obtidos em trabalhos anteriores realizados no Brasil ${ }^{(9-11)}$. Entretanto, concordamos que futuros estudos devem ser executados para elucidar definitivamente esta questão. Quando aplicamos os conceitos da classificação de Pirani et al ${ }^{(8)}$, devemos considerar, além do equinismo, a rigidez associada. Esta combinação, geralmente, determina um grau de maior severidade na avaliação inicial e que uma maior quantidade de aparelhos gessados devem ser utilizados.

Embora a efetividade do método de Ponseti $^{(2)}$ seja evidente, nos últimos 30 anos o papel da tenotomia do tendão calcâneo como elemento adjuvante da correção do PTC ainda não parece esclarecido. Esta tenotomia faz parte integrante desta abordagem terapêutica e está indicada em, aproximadamente, $70 \%$ a $80 \%$ dos pacientes.

De acordo com Hussain et $a l^{(12)}$, existem evidências que possam predizer se a tenotomia percutânea é necessária; porém, a maioria a realiza no pé deformado, especialmente quando o PTC idiopático é classificado como $\geq 5,0$ de Pirani et $a l^{(8)}$ ou grau IV de Diméglio et $a l^{(13)}$.

De acordo com algumas publicações, não há complicação relatada quando a correção cirúrgica do equinismo do retropé é realizada ${ }^{(4,14)}$. Entretanto, existem 
relatos da lesão da artéria fibular e da veia safena quando executada a tenotomia percutânea do tendão calcâneo. Anormalidades vasculares nos pacientes com PTC, como a hipoplasia ou agenesia da artéria tibial anterior ou da artéria tibial posterior, são circunstâncias bem documentadas na literatura $^{(4)}$. A ausência da artéria tibial posterior com concomitante insuficiência da tibial anterior determina um hiperfluxo da artéria fibular que a torna dominante nos pés acometidos e, nesta condição, a sua proteção torna-se imperiosa quando da execução da tenotomia percutânea ou liberação póstero-médiolateral. Sua lesão pode culminar em um membro isquêmico e, consequentemente, determinar uma amputação. O formato da lâmina utilizada também pode determinar risco de lesão ao nível da área tenotomizada, comprometendo a artéria tibial posterior, a veia safena parva e o nervo sural ${ }^{(3)}$.

Portanto, recomendamos que o exame do membro inferior operado seja realizado previamente, incluindo a palpação dos pulsos e complementada pela utilização do Doppler, especialmente nos casos em que há suspeita de agenesia ou hipoplasia arterial. Consequentemente, alguns autores defendem que a arteriografia poderia ser considerada para averiguação da predominância do suprimento circulatório do pé acometido na avaliação pré-operatória. Nestes casos, a abordagem aberta com visualização direta do tendão calcâneo deve ser considerada em busca da proteção direta da artéria fibular, diminuindo o risco da lesão iatrogênica desta estrutura anatômica.

Outro aspecto discutível e pouco pesquisado na área da ortopedia pediátrica relaciona-se ao local e ao tipo de anestesia aplicada para a realização da tenotomia. Seguindo os dogmas do método clássico, a mesma seria realizada no ambulatório após a aplicação de anestésico local. O procedimento poderia ser precedido pela anestesia tópica (lidocaína + prilocaína) realizada 30 minutos antes da execução do ato operatório evitando a punção local. Este procedimento pode ser considerado de risco e altamente estressante para os pais ou acompanhantes durante a realização da tenotomia, pois a criança apresenta choro copioso e agitação extremada. Existem os que realizam o procedimento no centro cirúrgico por considerarem este ambiente asséptico adequado para a realização da cirurgia, assim como mais seguro por garantir o efeito anestésico induzido pela técnica de anestesia geral, controle pelo monitor BIS $^{\circledR}$ (bispectral index monitor), haver monitorização, controle rigoroso de sangramento. Inclusive, o risco de acidente ocasionando ferimentos para o cirurgião e seus assistentes, mínimo risco de anafilaxia, convulsão, depressão miocárdica, toxicidade sistêmica, neuropatia periférica por injeção intraneural induzida pelo anestésico local, punção venosa inadvertida devem ser considerados $^{(15)}$. Contudo, este contexto demanda maior custo neste tempo operatório. A preocupação quanto à anafilaxia, broncoespasmos induzidos pelos anestésicos inalatórios determinando uma ação imediata, decorre exclusivamente do uso da anestesia geral. Na nossa amostra, a frequência de tenotomias realizadas, demonstrada na Tabela 3, evidencia que há concordância entre os resultados por nós observados com os encontrados na literatura $^{(16-21)}$. A maioria $(67,05 \%)$ dos médicos, como demonstrado na Tabela 4, realiza a operação no centro cirúrgico e não ambulatorialmente, pelos possíveis riscos inerentes á técnica cirúrgica, ocasionando uma lesão vascular complicada pelo sangramento ${ }^{(3)}$ e formação de pseudoaneurismas $^{(14)}$.

Os que realizam a tenotomia no ambulatório sugerem que a utilização de uma lâmina com extremidade romba poderiam reduzir esta potencial e devastadora complicação. Portanto, devemos estar atentos em perceber precocemente o dano circulatório no período pós-operatório. Outro fator a ser considerado relaciona o impacto socioeconômico do tratamento. $\mathrm{O}$ custo da tenotomia realizada no centro cirúrgico para o Sistema Único de Saúde do Brasil é de R\$284,06 (considerando um dia de internação para uma criança e seu acompanhante) e o custo da mesma realizada no ambulatório é de R\$22,06. Em um país subdesenvolvido como o Brasil, consideramos esta variável como muito importante, pois o procedimento ambulatorial atenua o custo.

Em relação aos resultados, observamos (Tabela 5) que a maioria obtém entre $50 \%$ e $80 \%$ de bons resultados com o método de Ponseti ${ }^{(2)}$. Este fato difere um pouco dos resultados obtidos pelo autor do método ${ }^{(16)}$, que relatou uma correção em torno de $90 \%$ dos casos e também com outros estudos ${ }^{(18-21)}$. Desta forma, estes resultados podem expressar a fase de aprendizado na aplicação do método de Ponseti ${ }^{(2)}$ pelos ortopedistas brasileiros, erros na aplicação do método ou viés estatístico quando nossos resultados são comparados com os de autores de outros países.

Apesar de altamente eficaz, este método cursa também com uma série de circunstâncias que propiciam os maus resultados e consistem basicamente de: manipulação inadequada, falha na utilização do aparelho ges- 
sado, aquiescência da utilização da órtese de abdução, correção incompleta e recidiva (Tabela 6).

Nosso estudo aponta que $98,7 \%$ dos ortopedistas orientam o uso do aparelho de abdução para a manutenção do resultado obtido. Destes, 67,5\% relatam que os familiares queixam-se da dificuldade em utilizar a órtese de Dennis-Brown, e esta questão ainda não está resolvida.

Com relação à recidiva das deformidades, observamos que esta ocorre com maior frequência em 10\% dos casos, concordando com os resultados encontrados em outros estudos ${ }^{(16-18)}$.

Uma adequada compreensão e aplicação da metodologia de Ponseti( ${ }^{(2)}$ permite que estas situações possam ser dirimidas. Diferenças nos graus de deformidade, inexistência de estudos controlados, variabilidade dos métodos de classificação para a avaliação inicial e final pós-tratamento, modificação dos métodos de tratamento de acordo com a experiência do cirurgião, adaptações de acordo com a disponibilidade de recursos dificultam que trabalhos científicos de melhor evidência sejam re-

\section{REFERÊNCIAS}

1. Kite $\mathrm{JH}$. Principles involved in the treatment of congenital club-foot. 1939. J Bone Joint Surg Am. 2003;85(9):1847.

2. Ponseti IV. The ponseti technique for correction of congenital clubfoot. J Bone Joint Surg Am. 2002;84(10):1889-90.

3. Minkowitz B, Finkelstein BI, Bleicher M. Percutaneous tendo-Achilles lengthening with a large-gauge needle: a modification of the Ponseti technique for correction of idiopathic clubfoot. J Foot Ankle Surg. 2004;43(4):263-5.

4. Dobbs MB, Gordon JE, Walton T, Schoenecker PL. Bleeding complications following percutaneous tendoachilles tenotomy in the treatment of clubfoot deformity. J Pediatr Orthop. 2004;24(4):353-7.

5. Rosner B. Fundamentos of biostatistics. 2nd edition. Boston: PWS Publishers; 1989. p. 584.

6. Lehman WB, Atar D, Grant AD, Strongwater AM. Functional rating system for evaluation of long-term results of clubfoot surgery. In: Simons GW, editor. The clubfoot: The present and a view of the future. New York: Springer-Verlag; 1994. p.114-6.

7. Bleck EE. Developmental orthopaedics. III: Toddlers. Dev Med Child Neurol. 1982;24(4):533-55.

8. Pirani S, Outerbridge H, Moran M, Sawatsky B. A method of evaluating the virgin clubfoot with substantial inter-observer reliability. J Pediatr Orthop. 1995;15:833-4.

9. Sodré H. Padronização do tratamento conservador do pé torto eqüinovaro congênito. Rev Bras Ortop. 1994;29(7):444-8.

10. Merllotti MH, Braga SR, Santili C. Pé torto congênito. Rev Bras Ortop. 2006;41(5):137-44. alizados. A avaliação acurada inicial pode prognosticar se há necessidade em se realizar a tenotomia do tendão calcâneo considerando conjuntamente a rigidez do retropé e o grau do equinismo. Portanto, acreditamos que a realização da tenotomia do tendão calcâneo, quando bem indicada, deve proporcionar bom resultado funcional. Apesar destes vieses, os princípios e objetivos do tratamento do PTC idiopático continuam inalterados, e a uniformização dos métodos de avaliação e tratamento permitiriam avaliações de maior valor científico além da utilização da randomização e de grupos controle.

\section{CONCLUSÕES}

Neste estudo, dos 5.329 ortopedistas participantes do $39^{\circ}$ CBOT, 539 foram entrevistados, 88 executam tratamento do PTC e, destes, 78 (88,60\%) têm como método preferencial para a sua realização o descrito por Ponseti. Observamos que $59(67,05 \%)$ dos ortopedistas que tratam PTC realizam a tenotomia do tendão calcâneo no centro cirúrgico, apesar da metodologia clássica de Ponseti defini-la como sendo ambulatorial.
11. Adames MK, Sodré H, Kuwajima SS. Protocolo clínico e radiográfico para avaliação de pacientes portadores de pé eqüinovaro congênito, após tratamento conservador. Rev Bras Ortop. 2001;36(6):205-12.

12. Hussain N, Khan T, Ahmed A. Complete subcutaneous tenotomy of tendoachilles in clubfoot patients - a four year follow up. J Surgery. 2004;2(1):17-9.

13. Diméglio A, Bensahel $H$, Souchet $P$, Mazeau P, Bonnet F. Classification of clubfoot. J Pediatr Orthop B. 1995;4(2):129-36.

14. Burghardt RD, Herzenberg JE, Ranade A. Pseudoaneurysm after Ponseti percutaneous Achilles tenotomy: a case report. J Pediatr Orthop. 2008;28(3):366-9.

15. Barash PG, Cullen BF, Stoelting RK. Clinical anesthesia. 5th edition. Philadelphia: Lippincott Williams \& Wilkins; 2005.

16. Ponseti IV, Smoley EN. Congenital club foot: the results of treatment. J Bone Joint Surg Am. 1963;45:261-344.

17. Laaveg SJ, Ponseti IV. Long-term results of treatment of congenital club foot. J Bone Joint Surg Am. 1980;62(1):23-31.

18. Tindall AJ, Steinlechner CW, Lavy CB, Mannion S, Mkandawire N. Results of manipulation of idiopathic clubfoot deformity in Malawi by orthopaedic clinical officers using the Ponseti method: a realistic alternative for the developing world? J Pediatr Orthop. 2005;25(5):627-9.

19. Göksan SB. [Treatment of congenital clubfoot with the Ponseti method]. Acta Orthop Traumatol Turc. 2002;36(4):281-7.

20. Segev E, Keret D, Lokiec F, Yavor A, Wientroub S, Ezra E, et al. Early experience with the Ponseti method for the treatment of congenital idiopathic clubfoot. Isr Med Assoc J. 2005;7(5):307-10.

21. Colburn M, Williams M. Evaluation of the treatment of idiopathic clubfoot by using the Ponseti method. J Foot Ankle Surg. 2003;42(5):259-67. 